\title{
The neural correlates of health risk perception in individuals with low and high numeracy
}

\author{
Stephan E. Vogel ${ }^{1}$ - Carmen Keller ${ }^{2} \cdot$ Karl Koschutnig $^{3} \cdot$ Gernot Reishofer $^{4}$. \\ Franz Ebner $^{4} \cdot$ Simone Dohle $^{2} \cdot$ Michael Siegrist $^{2} \cdot$ Roland H. Grabner $^{1}$
}

Accepted: 26 January 2016 / Published online: 9 February 2016

(C) The Author(s) 2016. This article is published with open access at Springerlink.com

\begin{abstract}
The ability to use numerical information in different contexts is a major goal of mathematics education. In health risk communication, outcomes of a medical condition are frequently expressed in probabilities. Difficulties to accurately represent probability information can result in unfavourable medical decisions. To support individuals with low-numeracy skills, pictographs such as graphs or icon arrays have been proposed to increase risk communication. The neurocognitive mechanism underlying the processing of health risk perception in individuals with lowand high-numeracy remain to be explored. To investigate the neural correlates of health risk perception, the brain responses of individuals with low- and high-numeracy were measured using functional magnetic resonance imaging. In the health risk perception condition, participants were instructed to indicate how concerned they would be of having cancer, given an icon array in which the probability of developing cancer was displayed. In the number line condition the same icon arrays were presented and participants were instructed to indicate the absolute number of displayed black icons. Results of the study showed that the low-numeracy group was more accurate in the health risk condition compared to the high-numeracy group, which
\end{abstract}

Stephan E. Vogel

stephan.vogel@uni-graz.at

1 Institute of Psychology, Educational Neuroscience, University of Graz, Universitätsplatz 2, 8010 Graz, Austria

2 Institute for Environmental Decisions, ETH Zürich, Universitätsstrasse 16, 8092 Zürich, Switzerland

3 Institute of Psychology, University of Graz, Universitätsplatz 2, $8010 \mathrm{Graz}$, Austria

4 Division of Neuroradiology, Medical University of Graz, Auenbruggerplatz 9, 8036 Graz, Austria overestimated health risks. A comparison of brain activation between the groups demonstrated that the high-numeracy group expressed larger brain engagement during the health risk condition in regions that are commonly associated with conflict monitoring, decision-making and emotional processing. These results provide initial evidence that individuals with high-numeracy engage regions of the brain to a different extent compared to individuals with low-numeracy.

Keywords Functional magnetic resonance imaging $(\mathrm{fMRI}) \cdot$ High- and low-numeracy $\cdot$ Number line $\cdot$ Health risk perception $\cdot$ Icon arrays

\section{Introduction}

The acquisition of numerical abilities is one of the pillars of mathematics education in enumerate societies. An increasing body of evidence has recently suggested that the development of basic numerical abilities (e.g., estimating the number of items in a set) builds an important foundation for arithmetic and mathematical competencies. For instance, it has been shown that individual differences in representing symbolic and non-symbolic numerical magnitudes are associated with individual differences in arithmetic competencies (e.g., Feigenson et al. 2013; Holloway and Ansari 2009). This suggests that the development of rich and accurate representations of numerical information (i.e., numeracy) is a crucial milestone on the road to mathematics. While there is growing evidence for a significant relationship between the development of numerical skills and arithmetic proficiency, our current understanding of how we process numerical information in different contextual situations (e.g., health risk decisions) to guide our behaviour and decisions 
is scarce. This is surprising, given that the flexible use of numerical information is a crucial cornerstone for the development of mathematical literacy, defined as "....an individual's capacity to formulate, employ, and interpret mathematics in a variety of contexts. It includes reasoning mathematically and using mathematical concepts, procedures, facts, and tools to describe, explain, and predict phenomena" (OECD 2012). The ability to accurately represent numbers and to flexibly use numerical information in different contexts is, therefore, an important goal towards which education and modern human societies strive. A better understanding of how individuals transfer and integrate numerical information into contexts in which adults are afforded to make important decisions is important to inform education practices and to foster the development of mathematical literacy.

Health decisions, in which chances and risks of a medical outcome have to be evaluated, are an emotionally charged context in which numerical information is used to make informed decisions about potential treatments. For instance, in hospitals patients are frequently faced with situations in which they have to evaluate chances and risks of a medical treatment. In order to allocate the necessary resources to counter a potential threat, a proper and accurate understanding of the involved odds and losses is indispensable. Hence, it is crucial that probabilities of a medical treatment are accurately perceived (Lipkus and Peters 2010). Current research has shown that a large number of individuals, particularly individuals with low numeracy skills, face severe difficulties to accurately understand numerical health risk information such as percentages, fractions and small probabilities (Gigerenzer and Edwards 2003; Lipkus and Peters 2009; Peters, 2008; Visschers et al. 2009). Therefore, a growing amount of evidence has reported unfavourable medical outcomes due to a lack of transferring numerical information to health risk contexts, particular in individuals with low-numeracy skills (e.g., Tanius et al. 2009; Zikmund-Fisher et al. 2008).

In the past, different solutions have been proposed to improve risk communication for individuals with lownumeracy skills (e.g., Gigerenzer and Edwards 2003). One of the discussed solutions is to express numerical information in form of visual non-symbolic numerical displays such as bar charts, risk ladders, scales or icon arrays (Apter et al. 2008; Gigerenzer and Edwards 2003; Hoffrage et al. 2000; Nelson et al. 2008), rather than with numerical symbols (i.e., Arabic digits). Icon arrays (also called pictographs) are visual non-symbolic representations of risk probabilities, which are displayed in form of faces, circles or other shaped icons (see Fig. 1). Specifically, the number of displayed icons expresses the frequency of people who are affected in contrast to the frequency of individuals who are unaffected by a medical condition (e.g., the probability of a medical condition).

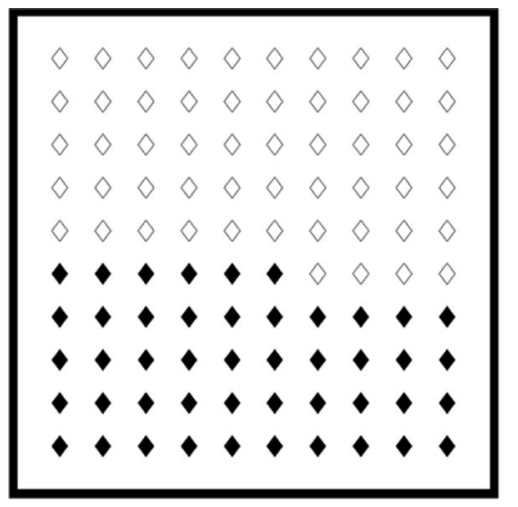

Fig. 1 Example of a canonical $10 \times 10$ icon array. This array contains 36 black and 64 white icons. The ratio between black and white icons displays the probability of a medical condition

Research into the effectiveness of icon arrays in health risk perception has shown that icon arrays improve numerical risk processing (Fagerlin et al. 2005; Feldman-Stewart et al. 2000; Galesic et al. 2009; Garcia-Retamero and Galesic 2010). Therefore, it has been argued that icon arrays are particular helpful in supporting individuals with low-numeracy skills to improve health risk communication. Accumulating evidence from behavioural studies indicates that the use of icon arrays increases accuracy (Galesic et al. 2009; Garcia-Retamero and Galesic 2010) and reduces unfavourable reasoning based on anecdotes of hypothetical treatment choices (Fagerlin et al. 2005). However, while the current evidence suggests an improvement in health risk communication using icon arrays, research into the underlying mechanisms of health risk perception is scarce.

A substantial body of literature exists on the neural correlates of risk processing in general (e.g., Herwig et al. 2011; Mohr et al. 2010). Economists and psychologists usually define risk as the uncertainty about which of several possible outcomes will occur, whereby the probability of each possible outcome is known (Knight 1921). Mohr et al. (2010) summarized previous findings in a meta-analysis and proposed a neurocognitive model of risk processing. In this model, three essential components are discussed that are engaged during risk processing. The first component of the model relates to emotional risk processing. Many decisions during risk evaluation are related to negative, disadvantageous or to a positive, advantageous outcome. Therefore, affective mechanisms have been found to exert influence on the cognitive evaluation during decision making and risk evaluation (Bach et al. 2009; Herwig et al. 2011; Quartz 2009; Vorhold et al. 2007; Xu et al. 2009). In line with this, consistent brain activation has been found in regions that are associated with emotional processing, such as the anterior insula and the thalamus (Mohr et al. 2010). The second component of the model relates to the cognitive 
evaluation of risks. At this stage, the probability of a particular outcome (e.g., of losses or chances) or the variance of a particular outcome is evaluated. Based on the results of the meta-analysis, the authors propose that the dorsomedial prefrontal cortex (DMPFC) plays a critical role in risk evaluation. This region of the brain has been found in studies that investigated response conflict, error monitoring, cognitive control and strategy selection (for reviews, see Ridderinkhof et al. 2004; Venkatraman et al. 2009). The last component of the risk model integrates emotional and cognitive information as well as other aspects of the stimuli, such as expected rewards, in order to form a final decision. This integrative component is proposed to be related to activation in the dorsolateral prefrontal cortex (DLPFC) and the parietal cortex; regions of the brain that are often found to be activated in decision making (for a review see Heekeren et al. 2008). The discussed evidence suggests that risk evaluation is an integrative mechanism in which cognitive components, such as processing numerical information (e.g., probabilities of an outcome), are integrated with affective components, which have been shown to exert an influence on cognitive risk evaluation. While some evidence indicates that individuals with low-numeracy express difficulties in processing the meaning of numerical information (e.g., probabilities) during health risk evaluation, there is also small evidence that individuals with lownumeracy skills draw less affective (i.e., emotional) meaning from numbers compared to their high-numeracy peers (Peters et al. 2006). Thus indicating that individuals with low-numeracy skills may not only face difficulties in evaluating numerical information but have also difficulties in processing the affective meaning of numerically presented risk information. However, little is currently known how cognitive and affective components interact in health risk perception and how such mechanisms may differ between individuals with high- and low-numeracy.

The aim of the present functional Magnetic Resonance Imaging (fMRI) study was to investigate the brain regions involved in health risk perception to better understand the cognitive and affective mechanisms that underlie health risk perception, and how such mechanisms may differ between individuals with high- and low-numeracy skills. We asked participants to imagine a hypothetical situation in which they have undergone a medical screening for colon cancer. Using similar icon arrays as in previous studies (e.g., Galesic et al. 2009; Hess et al. 2011), individuals of a low- and high-numeracy group were asked to indicate their concern of having cancer on an analogue physical number line, ranging from 0 on the left to 100 on the right (health risk perception condition). In an additional experimental condition (number line condition), the same icon arrays were presented, but individuals were only required to estimate the total number of filled (black) icons and to indicate the value on an analogue scale, ranging from 0 on the left to 100 on the right.

Based on the reviewed literature we expected to find a larger engagement of brain regions that are commonly found in risk processing. Since risk evaluation affords $\operatorname{cog}$ nitive demanding operations, such as reflecting on numerical information, we expected larger brain activation in regions that are associated with cognitive control, strategy selection and numerical information processing-such as the dorsomedial prefrontal cortex and inferior parietal cortex. Moreover, we speculated that differences in brain activation between the two numeracy groups emerge on the level of cognitive operation and/or affective processing. More specifically, if individuals with low-numeracy show difficulties in processing numerical information, reduced brain activation in regions of the inferior parietal cortex (e.g., Kaufmann et al. 2009; Price et al. 2007) may be expected. However, if individuals with low-numeracy express particular difficulties in charging the presented numerical information with affective valence, reduced brain activation may be found in regions that are associated with emotional processing such as the thalamus and the insula (Mohr et al. 2010; Peters et al. 2006).

\section{Methods}

\subsection{Participants}

Thirty-eight right-handed healthy adults participated in the present functional neuroimaging study (18 men, 20 female; mean age 23.49 years, range 18-32 years). The group comprised individuals with normal or corrected to normal vision, and all participants reported German to be their native language. Individuals received a monetary compensation and gave written informed consent. The local Ethics Board at the Medical University of Graz approved the study.

Participants were assigned to a "high-numeracy group" and to a "low-numeracy group". Group allocation was based on the results of the Subjective Numeracy Scale (SNS; Fagerlin et al. 2007). The SNS is an 8-item questionnaire that measures individuals' perceived mathematics abilities and their subjective preference to represent numerical information. Half of the items assess the perceived ability to perform numerical tasks in various contexts (i.e., SNS ability subscale). An example item of the SNS ability subscale is: "How good are you at working with fractions?" The second half of the questionnaire measures the subjective preference to represent probabilistic or numerical information (i.e., SNS preference subscale). An example item of the SNS preference subscale is: "When reading the newspaper, how helpful do you find tables and graphs 
that are parts of a story?" Participants rated the 8 questions on a 6 point Likert-Scale from " $1=$ not good at all/not helpful at all" to " $6=$ extremely good/extremely helpful". Individuals' ratings were aggregated to a mean SNS score. The SNS was developed as an easy and quick tool to predict people's objective numerical abilities. The scale was evaluated in a set of different studies and has demonstrated a high reliability (Cronbach's alpha 0.82) (Fagerlin et al. 2007; Zikmund-Fisher et al. 2007). Moreover the SNS has been shown to significantly correlate $(r=0.68, p<0.001)$ with the "Lipkus and Others' Objective Numeracy Scale", which assesses people's ability to perform mathematical tasks on risk magnitudes using percentages and proportions as well as their ability to convert percentages to proportions, proportions to percentages and probabilities to proportions (Fagerlin et al. 2007; Lipkus et al. 2001; ZikmundFisher et al. 2007).

Based on the individual mean SNS scores, a median split was calculated and half of the participants (5 men, 14 women; mean age 22.83 years; range 18-32 years) were assigned to a "low-numeracy group" (mean SNS score 3.55, SD 0.52), while the others (13 men, 6 women; mean age 24.11 years; range 19-31 years) were assigned to a "high-numeracy group" (mean SNS score 4.78, $\mathrm{SD}=0.33$.

\subsection{Task design and stimuli}

Individuals' behavioural responses and brain activations were recorded during two different task conditions: a nonsymbolic "Health Risk Perception" (HRP) task and a nonsymbolic "Number Line" (NL) approximation task.

In the HRP condition, participants were required to imagine that they had undergone a screening test for colon cancer and that the doctor informs them about the screening results by means of pictographs. The pictographs comprised specific ratios of filled and empty icons, which indicated the probability (1-99\%) of having cancer. Based on this information participants were instructed to indicate how concerned (from "not at all concerned" to "very concerned") they would be of having cancer. Participants used a fiber optic trackball response pad (Current Designs Inc., Pennsylvania, USA) to indicate their concerns by moving a red arrow to the desired location on the scale and by confirming the rating by a button-press.

In the NL condition participants were presented with the same non-symbolic pictographs as in the HRP condition. However, in this task participants were explicitly instructed to estimate the total number of filled icons and to indicate this value on an analog scale (ranging from 1 to 99). Participants used the same trackball device to execute their decisions. Please note that stimuli (i.e., pictographs and the analog number line) were identical in both conditions. The only difference between the HRP and NL condition was the task instruction.

The pictographs comprised 100 diamond shaped icons, arranged in a $10 \times 10$ matrix displayed on a white square. The ratio between filled and empty icons represented numerical information upon which individuals had to base their decisions. In the HRP condition, the ratio between the filled and empty icons displayed the probability (between 1 and $100 \%$ ) of having colon cancer. In the NL conditions, the black diamonds displayed a numerical value between 1 and 100 (see Fig. 2).

In addition to the pictograph, a horizontal analog number line was presented on the computer screen. Two landmarks, one at the left and one at the right corner, supplemented the number line. In the NL condition, the left landmark represented the numerical value 0 , while the right landmark represented the numerical value 100 . In the HRP condition, the landmarks indicated concerns of having cancer. The left landmark represented "not at all concerned", while the right represented "very concerned". On every trial the analog number line, on which participants indicated their judgements/estimations, appeared simultaneously with the pictograph.

A total number of 60 trials (i.e., pictographs and number line) were presented. The 60 trials comprised six randomly selected numbers from each decade (10 decades $\times 6$ numbers $=60$ in total). These preselected numbers were combined into a pseudo-randomized sequence and presented in the same order to all participants. Landmark numbers (e.g., 25, 50, 75) as well as decade numbers (i.e., 10, 20...90) were excluded to avoid alternative strategies (e.g., simply dividing the number line when the number 50 is presented; Vogel et al. 2013). Half of the preselected trials contained even number items, while the other half contained odd number items. No number from the same decade was displayed on subsequent trials (see "Appendix"). The same stimuli selection criteria were applied for the NL condition. In contrast to the HRP condition a total number of 40 number trials over two functional runs were used. ${ }^{1}$ In this case, 4 numbers were randomly preselected from each decade $(10$ decades $\times 4$ numbers $=40$ in total).

\footnotetext{
${ }^{1}$ In the same study, a second NL condition with numerical symbols was administered for investigating a different research question. The results of this additional condition are not reported in the present study. Given a finite number of numerals within a decade, only 4 trials of every decade could be used in the non-symbolic condition and in the symbolic condition without resulting in a number overlap. As a consequence the total number of trials in the NL condition of the present study is restricted to 40 .
} 


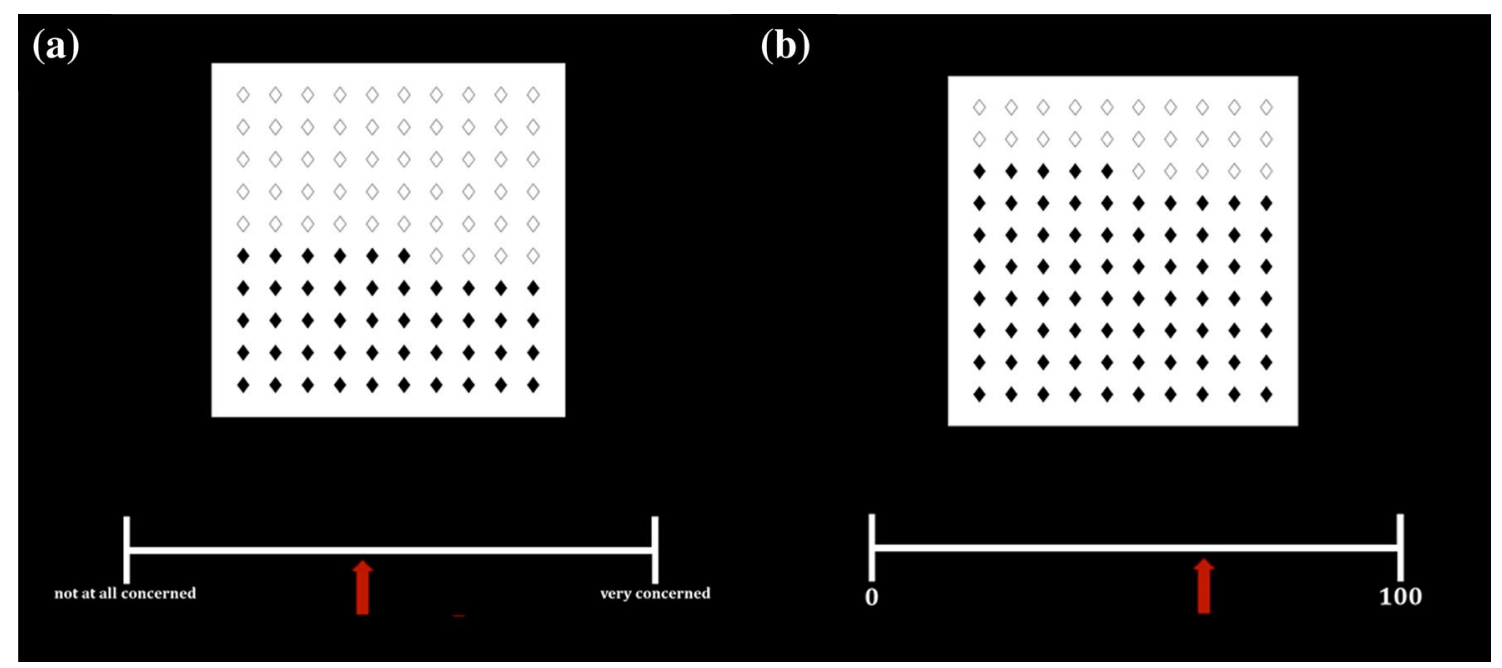

Fig. 2 Example stimuli of the a HRP and b NL condition. The number of filled icons (46 on the left and 75 on the right) exemplify numerical values in the NL condition and probability values of hav- ing cancer in the HRP condition. The red arrow on the number line was moved by trackball device with which participants had to indicate their decision based on the task instruction (color figure online)

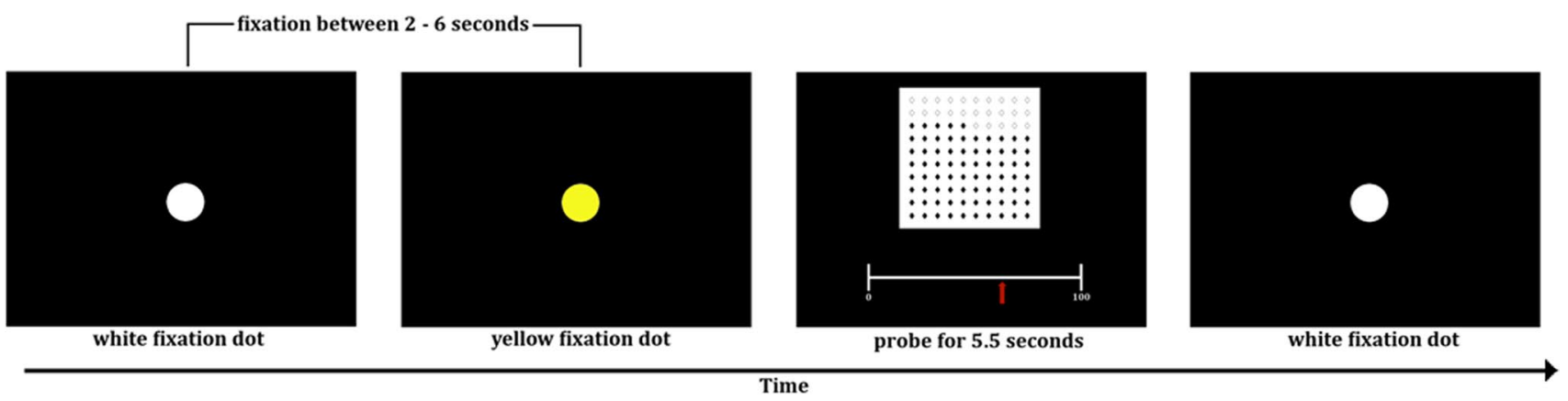

Fig. 3 Illustration of one experimental trial of the NL condition. See Tables 1 and 2 for a complete list of the numerical stimuli used

\subsection{Experimental procedure}

Participants were familiarized with the fMRI environment prior to scanning. In order to minimize participants' head motion during data acquisition, soft paddings were placed between participants' head and the scanner head-coil. Once positioned in the scanner, participants performed four functional runs-two runs of the HRP and two of the NL condition. Please note that all participants performed the HRP condition prior to the NL condition, since a potential influence on strategy may occur if participants performed the NL condition prior to the HRP condition.

Each trial (see Fig. 3) started with a fixation period between 2 to $6 \mathrm{~s}$ (average $4 \mathrm{~s}$ ). During this fixation period a white dot appeared in the center of the computer screen, followed by a yellow dot. Participants were instructed to fixate the dot at all times and to prepare for the next trial once the white dot has turned into a yellow dot. After this fixation period, the stimuli appeared for $5.5 \mathrm{~s}$ on the computer screen. In every trial a novel pictograph was presented, which contained hypothetical information about the probability of having cancer in the HRP condition, or the total number of filled icons in the NL condition. Participants were then required to make a manual response by moving a little red arrow from its starting position to the desired location on the number line. The starting position of the red arrow was randomized in such away that the numerical distance between the "correct" number position and the starting position on the number line was at least 10 numerical values apart (see "Appendix"). After $5.5 \mathrm{~s}$ the stimuli probe disappeared and the next trial started with a white fixation dot on the screen. Please note that a pseudo randomized jitter between 2 to $6 \mathrm{~s}$ was interspersed between the probe and the fixation period to ensure oversampling and a better estimation of the Hemodynamic Response Function (HRF). 
Stimuli presentation and behavioural response recording were executed by the presentation software package Presentation ${ }^{\circledR} 14.8$ (Neurobehavioral Systems Inc., Berkeley, CA, USA). All stimuli were presented on to a diffusing screen outside the magnet bore using a mounted LCD projector.

\subsection{MRI data acquisition}

Data collection was done with a whole body 3T-Siemens Tim Trio MRI scanner. A single shot gradient echo (ss-EPI) $\mathrm{T}^{*}$ weighted sequence (time of repetition $(\mathrm{TR})=2000 \mathrm{~ms}$; echo time $(\mathrm{TE})=25 \mathrm{~ms}$; flip angle $=90^{\circ}$ ) in combination with a 32-channel head-coil was used to measure changes in Blood Oxygen Level Dependent (BOLD) signal. Each functional session comprised 338 whole-brain volumes that were collected in a descending-interleaved acquisition order. One brain volume consisted of 34 slices $(3 \mathrm{~mm}$; matrix size $=64 \times 64$ with a distance factor of 0.75 ) resulting in voxel resolution of $3 \mathrm{~mm} \times 3 \mathrm{~mm} \times 3.75 \mathrm{~mm}$. To account for T1 saturation, the first two volumes of every functional run were automatically discarded. High-resolution structural images were acquired with a T1-weighted 3D MPRAGE sequence (176 slices, TR $=1900 \mathrm{~ms}$; TE $=2.19 \mathrm{~ms}$; flip angle $=9^{\circ}$; inversion time $=900 \mathrm{~ms} ; \mathrm{FOV}=256 \times 256$ ), resulting in an isotropic $1 \mathrm{~mm} \times 1 \mathrm{~mm} \times 1 \mathrm{~mm}$ voxel resolution.

\subsection{Behavioural analysis}

For every individual a mean deviation score was calculated for the NL and the HRP condition respectively. Mean deviation scores were measured as positive (to the right) or negative (to the left) response deflections from the "correct" position on the number line for a given number trial. For instance, if a participant placed the arrow 2 values to the left of the "correct" position, a value of -2 would have been scored for that particular trial. Individual mean deviation scores were calculated for the HRP and the NL condition and entered into a $2 \times 2$ repeated measure analysis of variance (ANOVA) with Condition (HRP vs. NL) as within subject factor and Group (high-numeracy vs. low-numeracy) as between subject factor.

\section{6 fMRI data analysis}

The acquired functional images were pre-processed using the brain imaging analysis software package SPM5 (Wellcome Department of Imaging Neuroscience, London, UK). Individual runs were motion- and slice-time corrected, coregistered with the structural data and spatially normalized into MNI space (Montreal Neurological Institute). A 8 mm Full-Width-of-Half Maximum (FWHM) Gaussian smoothing kernel was applied, and a temporal high-pass filter of $1 / 256 \mathrm{~Hz}$ to remove low frequency signals (Huettel et al. 2008).

A Random Effects (RFX) General Linear Model (GLM) was calculated to investigate brain activations. To investigate the brain activation particularly associated with the HRP condition, the first contrasts pitted the brain activation of the HRP task against the brain activation of the NL task for each group separately (i.e., high- and low-numeracy). In other words, we performed a statistical comparison of the contrast "HRP > NL" in the high-numeracy group, and a statistical comparison of the contrast "HRP $>$ NL" in the low-numeracy group. In order to investigate brain activation differences between the groups, a t-contrast was calculated pitting the brain activation of the low-numeracy group against the brain activation of the high-numeracy group (i.e., [high-numeracy "HRP $>$ NL"] $>$ [low-numeracy "HRP > NL"]).

\section{Results}

\subsection{Behavioural results}

The $2 \times 2$ ANOVA of the deviation scores revealed a significant main effect of Condition $(F(1,36)=10.737$; $\left.p=0.002 ; \eta^{2}=0.230\right)$ and a significant main effect of $\operatorname{Group}\left(F(1,36)=10.791 ; p=0.002 ; \eta^{2}=0.231\right)$. Behavioural responses were more accurate in the NL condition (mean deviation scores $=-1.594$ ) compared to the HRP condition (mean deviation scores $=2.207$ ); the "lownumeracy group" (mean deviation scores $=-1.990$ ) was more accurate than the "high-numeracy group" (mean deviation scores $=2.603$ ). In addition to these main effects the ANOVA revealed a significant interaction between Condition $\times$ Group $\left(F(1,36)=5.118 ; p=0.03 ; \eta^{2}=0.124\right)$, indicating significant group differences in the way individuals responded in the HRP and NL condition. To further explore this interaction statistically, two paired-sample t-tests were calculated, which contrasted the mean deviations scores of the HRP condition against the mean deviations scores of the NL condition for both groups separately. Results of the two t-tests demonstrated a significant difference between the mean deviation scores of the HRP and NL condition in the high-numeracy group $\left(t_{19}\right)=3.545$; $p=0.002)$, whereas no significant difference was found in the low-numeracy group $\left(t\left(_{19}\right)=0.813 ; p=0.427\right)$. To investigate differences between the groups, two additional independent-sample t-tests were performed for the HRP and the NL condition separately. Results of these analyses showed a significant difference between the high-numeracy group and the low-numeracy group in the NL condition $\left.\left(t{ }_{19}\right)=2.629 ; p=0.013\right)$, as well as in the HRP condition 


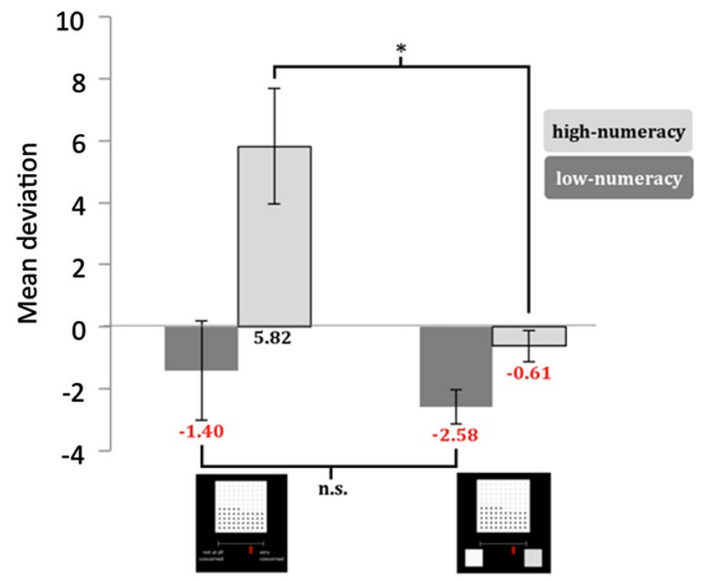

Fig. 4 Mean deviation scores of the HRP and NL condition as a function of "high-numeracy group" and "low-numeracy group"

$\left.\left(t{ }_{19}\right)=2.937 ; p=0.006\right)$. Together, these results indicate that behavioural responses varied as a function of the task but also as a function of numeracy (see also Figs. 4, 5).

\subsection{Imaging results}

The first contrast of the neuroimaging analyses aimed to identify those brain regions that showed larger brain activation in the HRP condition compared to the NL condition. Within the "high-numeracy group", the results of this contrast revealed a set of brain regions that showed significant greater brain activation in the HRP condition compared to the NL condition (see Table 1). In particular, brain regions of the right fronto-parietal network (see Fig. 6) were engaged to a larger extent in the HRP condition compared to the NL condition.

Results for the same contrast in the "low-numeracy group" revealed a smaller set of brain regions (see Table 2), which demonstrated an activation difference between the HRP and the NL condition. Nevertheless, similarly to the "high-numeracy group" activation differences were primarily found in brain regions of the right fronto-parietal network (see Fig. 7).

The final contrast of the present study aimed to statistically compare the brain activation of the contrast (HRP > NL) between the "high-numeracy group" and the "low-numeracy group". While no significant cluster was revealed at a statistical threshold level of $\mathrm{p}<0.001$ uncorrected (FWE cluster corrected $\mathrm{p}<0.05$ ), four brain regions showed a larger brain activation in the "high-numeracy group" compared to the "low-numeracy group" when the statistical threshold was lowered to $\mathrm{p}<0.001$ uncorrected (minimum cluster size $=10$ voxels). Among these regions were the anterior cingulate, the right precuneus and the thalamus (see Table 3; Fig. 8). The reverse contrast (i.e., larger brain activation for the "low-numeracy group" compared to the "high-numeracy group") did not reveal brain activation differences between the groups.

\section{Discussion}

The mastery of numerical abilities is crucial in modern human societies. While an extensive body of research has demonstrated a significant association between individual differences in numerical skills and mathematical competencies (Feigenson et al. 2013; Holloway and Ansari 2009), less is currently known about how we use numerical information in different contextual situations in order to evaluate potential consequences of our behaviour. This is surprising, given that the flexible use of numerical information is crucial in order to describe, explain and predict different phenomena (OECD 2012). Deepening our understanding of how we translate and integrate numerical information during decision-making may have profound implications for education. Thus the present study aimed to investigate the cognitive and affective mechanisms involved during health risk perception and evaluation.

In line with our predictions, the imaging results of the present study demonstrated significant larger brain activation during health risk perception in regions of the medial frontal cortex, encompassing regions of the DLPFC and DMPFC, the parietal cortex as well as in regions that are associated with emotional processing such as the insula and thalamus. Previous research has shown that the DLPFC, DMPFC, and the parietal cortex are frequently engaged in risk processing (e.g., Herwig et al. 2011; Mohr et al. 2010). Modulation of brain activation in these regions has been related to executive functions such as response conflict, error monitoring, cognitive control, strategy selection and decision making (Heekeren et al. 2008; Ridderinkhof et al. 2004; Venkatraman et al. 2009). Besides brain activation in frontal regions, larger brain activation was also found in the parietal cortex. The parietal lobe of the human brain has not only been implicated in risk processing (Mohr et al. 2010), but has also been shown to play a crucial role in numerical information processing (Ansari and Vogel 2013; Ansari 2008; Dehaene et al. 2003; Nieder and Dehaene 2009; Vogel and Ansari 2012). To date a large body of evidence has demonstrated that the intraparietal sulcus (IPS) of the parietal lobe is consistently engaged whenever numerical magnitudes are processed. For instance, modulation of brain activation in the parietal lobe has been found in number comparison (Ansari et al. 2005; Holloway et al. 2010; Pinel et al. 1999, 2001), in the context of calculation (e.g., Grabner et al. 2007; Simon et al. 2002, 2004), in tasks in which the spatial position of a given number on a physical number line has to be estimated (Vogel et al. 2013) and in 


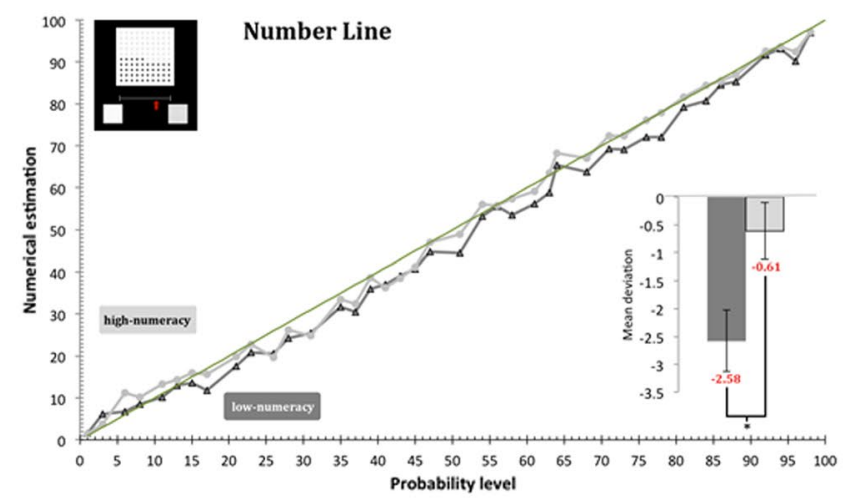

Fig. 5 The graphs depict the mean deviation scores for single number trials presented to the participants for the NL (on the left) and for the HRP (on the right) condition respectively. The diagonal line in both displays denotes the objective "correct" position of the presented number trial. The dark gray line represents mean deviation scores

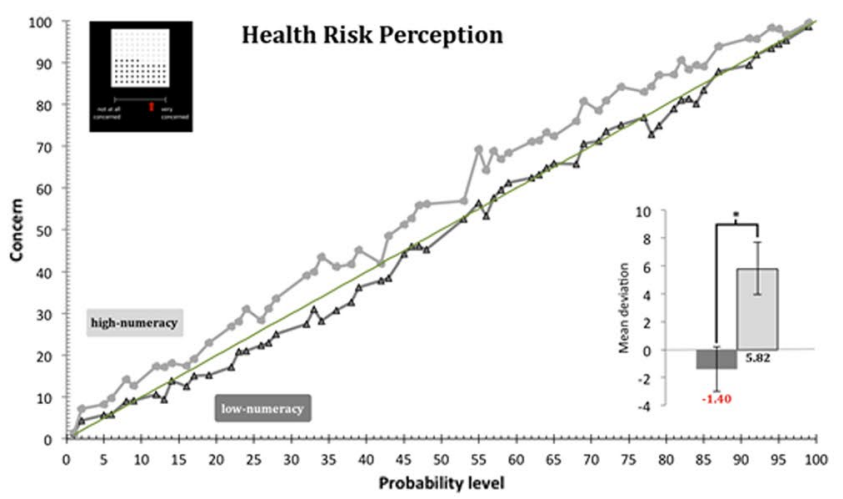

from the main diagonal line of the "low-numeracy group", the bright gray line represents mean deviations scores from the diagonal line of the "high-numeracy group". Bar graphs depict the average mean deviation scores for the "low-numeracy group" and the "high-numeracy group"

Table 1 Brain regions of the "high-numeracy group" that showed larger activation in the HRP condition compared to the NL condition

\begin{tabular}{|c|c|c|c|c|c|c|c|}
\hline \multirow[t]{2}{*}{ Region } & \multirow[t]{2}{*}{ Hemisphere } & \multicolumn{3}{|c|}{ MNI coordinates } & \multirow[t]{2}{*}{ \#of voxels } & \multirow{2}{*}{$\begin{array}{l}\mathrm{p}(\mathrm{FWEc} \\
\text { corrected) }\end{array}$} & \multirow{2}{*}{$\begin{array}{l}\text { Peak } \\
\text { intensity }\end{array}$} \\
\hline & & $\mathrm{x}$ & $\mathrm{y}$ & $\mathrm{z}$ & & & \\
\hline Sub-gyral (frontal) & $\mathrm{L}$ & -18 & 3 & 27 & 416 & 0.001 & 8.27 \\
\hline Superior frontal g. & $\mathrm{R}$ & 36 & 48 & -15 & 762 & 0.001 & 7.73 \\
\hline Declive & $\mathrm{R}$ & 18 & -75 & -18 & 1019 & 0.001 & 7.73 \\
\hline Inferior parietal 1. & $\mathrm{R}$ & 51 & -48 & 54 & 903 & 0.001 & 7.56 \\
\hline Insula & $\mathrm{L}$ & -39 & 12 & 0 & 216 & 0.001 & 7.08 \\
\hline Sub-gyral (temporal) & $\mathrm{L}$ & -36 & -33 & -3 & 126 & 0.001 & 6.82 \\
\hline Middle frontal g. & $\mathrm{R}$ & 36 & 27 & 48 & 308 & 0.001 & 6.67 \\
\hline Precuneus & $\mathrm{R}$ & 3 & -60 & 63 & 46 & 0.020 & 6.61 \\
\hline Postcentral g. & $\mathrm{L}$ & -33 & -39 & 69 & 71 & 0.002 & 6.00 \\
\hline Thalamus & $\mathrm{R}$ & 3 & -18 & 12 & 181 & 0.001 & 5.98 \\
\hline Postcentral g. & $\mathrm{L}$ & -45 & -24 & 39 & 103 & 0.001 & 5.46 \\
\hline Superior frontal g. & $\mathrm{L}$ & -30 & 48 & 27 & 79 & 0.001 & 5.42 \\
\hline Parahippocampal g. & $\mathrm{L}$ & -33 & -6 & -18 & 40 & 0.037 & 5.37 \\
\hline Precuneus & $\mathrm{R}$ & 15 & -57 & 39 & 58 & 0.007 & 5.11 \\
\hline Middle temporal g. & $\mathrm{R}$ & 57 & -30 & -15 & 84 & 0.001 & 5.06 \\
\hline
\end{tabular}

Brain regions are sorted by peak intensity; regions were interpreted as significant if intensity values passed a threshold of $\mathrm{p}<0.001$ uncorrected (FWE cluster corrected $\mathrm{p}<0.05$ )

fMRI-Adaptation studies in which the neural signal recovery in response to numerical deviants has been investigated (Holloway et al. 2012; Vogel et al. 2015). Since risk evaluation is a cognitively demanding operation, participants are afforded to reflect upon numerical information in order to estimate their subjective health concerns, the greater modulation of brain activation in the parietal lobe, particular the IPS, may be explained by a larger engagement of numerical information processing during health risk evaluation than during number line estimation.

When contrasting the brain activation between individuals with high- and low-numeracy, differences in brain activation were found in bilateral regions of the anterior cingulate, the thalamus and the right precuneus. These regions were engaged to a greater extent in individuals with high-numeracy in contrast to individuals with low-numeracy (i.e., [high-numeracy "HRP > NL"] > [low-numeracy "HRP $>$ NL"]). ${ }^{2}$ Modulation of brain activity in the anterior

\footnotetext{
${ }^{2}$ Post-hoc independent sample t-tests demonstrated no differences in reaction times between the low- and high-numeracy group, neither in the MNL $\left(\mathrm{t}\left({ }_{36}\right)=-0.186 ; \mathrm{p}<0.854\right)$ nor the HRP $\left(\mathrm{t}\left({ }_{36}\right)=1.329\right.$; $\mathrm{p}<0.192)$ condition. This indicates that differences in cognitive effort do not explain the observed activation differences between the two groups.
} 
Fig. 6 Brain activation difference between the HRP and NL condition in the "high-numeracy group". Significant brain activation clusters are displayed on the surface of an inflated brain ( $A$ anterior, $P$ posterior)

\section{High-numeracy group HRP > NL}

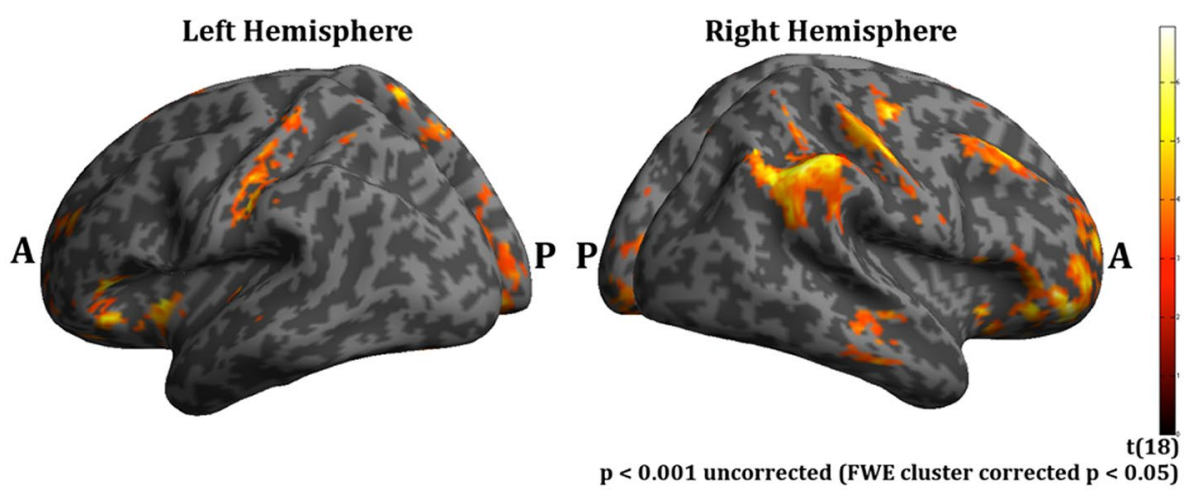

Table 2 Brain regions of the "low-numeracy group" that showed a larger activation in the HRP condition compared to the NL condition

Fig. 7 Brain activation difference between the HRP condition and NL condition in the "low-numeracy group". Significant brain activation clusters are displayed on the surface of an inflated brain ( $A$ anterior, $P$ posterior)

Table 3 List of those brain regions that showed a larger activation in the "highnumeracy group" compared the "low-numeracy group"

\begin{tabular}{|c|c|c|c|c|c|c|c|}
\hline \multirow[t]{2}{*}{ Region } & \multirow[t]{2}{*}{ Hemisphere } & \multicolumn{3}{|c|}{ MNI coordinates } & \multirow[t]{2}{*}{ \#of voxels } & \multirow{2}{*}{$\begin{array}{l}\mathrm{p}(\mathrm{FWEc} \\
\text { corrected })\end{array}$} & \multirow{2}{*}{$\begin{array}{l}\text { Peak } \\
\text { intensity }\end{array}$} \\
\hline & & $\mathrm{x}$ & $\mathrm{y}$ & $\mathrm{z}$ & & & \\
\hline Middle frontal g. & $\mathrm{R}$ & 45 & 48 & -12 & 130 & 0.001 & 6.55 \\
\hline Inferior parietal 1. & $\mathrm{R}$ & 57 & -54 & 39 & 422 & 0.001 & 6.24 \\
\hline Lingual g. & $\mathrm{R}$ & 12 & -84 & -15 & 266 & 0.001 & 5.78 \\
\hline Middle frontal $\mathrm{g}$. & $\mathrm{L}$ & -21 & 57 & 24 & 42 & 0.046 & 4.80 \\
\hline
\end{tabular}

Regions are listed by peak intensity; activation clusters were interpreted as significant if the passed a threshold of $p<0.001$ uncorrected (FWE cluster corrected $p<0.05$ )

\section{Low-numeracy group HRP > NL}
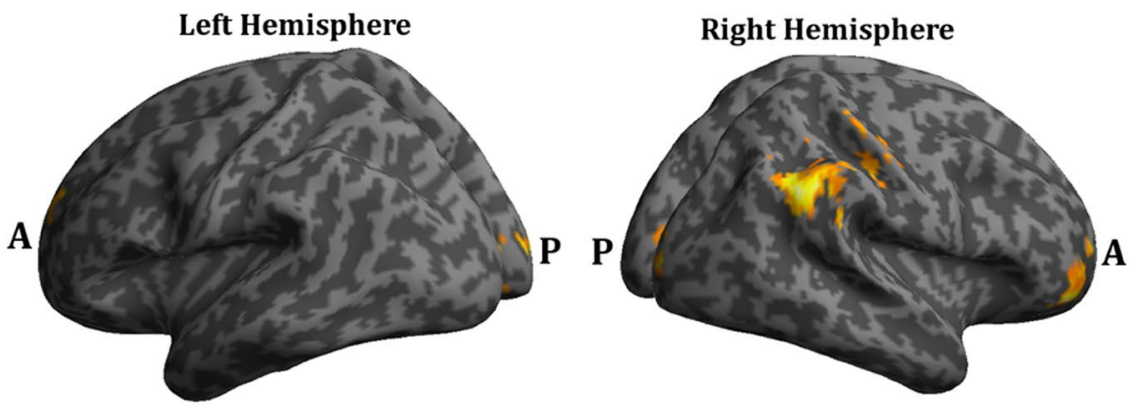

$\mathrm{p}<0.001$ uncorrected (FWE cluster corrected $\mathrm{p}<0.05$ )

\begin{tabular}{|c|c|c|c|c|c|c|c|}
\hline \multirow[t]{2}{*}{ Region } & \multirow[t]{2}{*}{ Hemisphere } & \multicolumn{3}{|c|}{ MNI coordinates } & \multirow[t]{2}{*}{ \# of voxels } & \multirow{2}{*}{$\begin{array}{l}\mathrm{p}(\text { uncorrected }) \\
\text { Voxel size }>10\end{array}$} & \multirow{2}{*}{$\begin{array}{l}\text { Peak } \\
\text { intensity }\end{array}$} \\
\hline & & $\mathrm{x}$ & $\mathrm{y}$ & $\mathrm{z}$ & & & \\
\hline Anterior cingulate & $\mathrm{R}$ & 18 & 27 & 21 & 21 & 0.001 & 4.56 \\
\hline Precuneus & $\mathrm{R}$ & 18 & -54 & 42 & 12 & 0.001 & 4.47 \\
\hline Anterior cingulate & $\mathrm{L}$ & -6 & 42 & 0 & 11 & 0.001 & 4.24 \\
\hline Thalamus & Inter & 0 & 15 & 12 & 11 & 0.001 & 4.00 \\
\hline
\end{tabular}

Significance threshold was set to $\mathrm{p}<0.001$ uncorrected (minimum cluster size $=10$ voxels) 

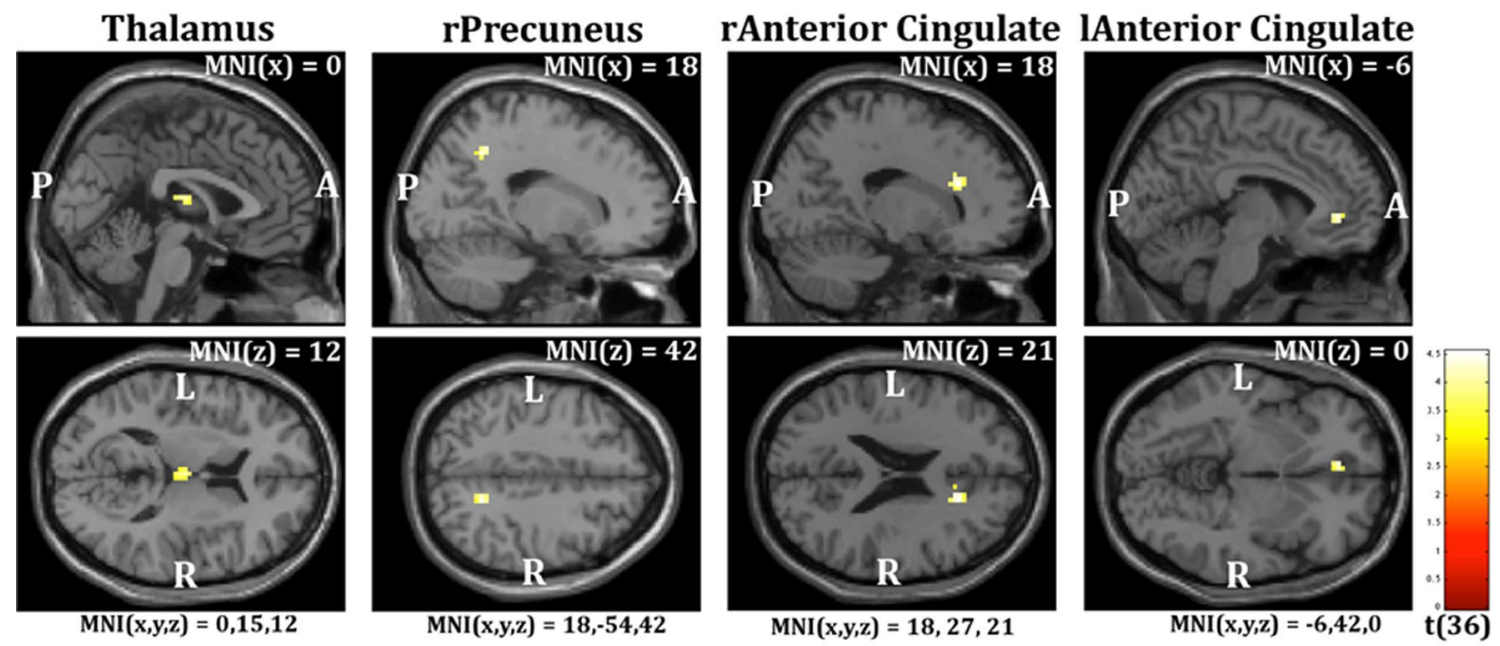

$\mathrm{p}<\mathbf{0 . 0 0 1}$ uncorrected (minimum culster size $=\mathbf{1 0}$ voxel)

Fig. 8 Sagittal and transversal view of those regions that showed greater brain activation in the "high-numeracy group" compared to the "lownumeracy group" ( $R$ right, $L$ left, $A$ anterior, $P$ posterior)

cingulate and thalamus has been previously reported during risk processing (Bach et al. 2009; Herwig et al. 2011; Mohr et al. 2010; Quartz 2009; Vorhold et al. 2007; Xu et al. 2009). Specifically, it has been argued that modulation of these brain regions is reflective of emotional processing during risk evaluation (e.g., Herwig et al. 2011; Mohr et al. 2010). For instance, a study by Herwig et al. (2011) investigated the neural correlates associated with the evaluation of societal hazards in a group of 20 healthy adult participants. In this fMRI study, participants were instructed to evaluate 50 more or less societal hazard statements presented as written terms on a computer screen. Results of the brain imaging analyses revealed that statements, which were rated as high societal risks (e.g., smoking), elicited greater brain activation in the medial thalamus, anterior insula, caudate nucleus and cingulate cortex in comparison to statements that were rated as low societal risks (e.g., food conservation). The authors interpreted the greater engagement of the cingulate, the anterior insula and the caudate nucleus as evidence for a larger involvement of emotional processing (e.g., viscerosensitive signals) during the highrisks evaluation, which may be interpreted as an intuitive "gut-feeling" towards risky conditions. Although speculative, the observed group differences in brain activation in the bilateral anterior cingulate and thalamus may be seen as differences in emotional processing between individuals with high-numeracy and individuals with low-numeracy during health risk perception. The greater activation of these regions in individuals with high-numeracy, may indicate a larger emotional involvement (e.g., gut feeling) during health risk perception compared to individuals with low-numeracy. This interpretation is also in line with previous behavioural findings that have indicated that individuals with low-numeracy may charge numerical information with different emotional valence and perceive health risks differentially compared to their high-numeracy peers (Peters et al. 2006).

The imaging results also revealed a greater engagement of the precuneus in individual with high-numeracy skills. The precuneus is anatomically connected to subcortical structures and to association regions of the cortex, suggesting a pivotal role of the precuneus in an extensive network concerned with the elaboration and integration of associative information. Additional evidence from neuroimaging studies has indicated that the precuneus is involved in visuo-spatial imagery, episodic memory retrieval, selfprocessing operations and consciousness (for a review see Cavanna and Trimble 2006). In addition, the precuneus has recently proposed to be part of the parietal memory network (PMN), which shows modulation in brain activation depending on how strongly a stimuli captures one's attention (Gilmore et al. 2015). In light of this evidence the observed activation differences in the present study may be explained by differences in the degree to which external representations of health risk probabilities capture one's attention and are mentally integrated into internal subjective health risk perceptions. Specifically, in the HRP condition participants were asked to express their health risk concerns based on external representations of visuo-spatial icon arrays. In order to evaluate health risks concerns, individuals with high-numeracy skill may be more attentive towards numerical information in order to integrate the visuo-spatial information into mental images of health risk concerns. However, it should be noted that the functional 
significance of the precuneus remains a matter of debate and that its role in the present study is speculative. Clearly more evidence is needed to better understand the engagement of the precuneus during health risk evaluation.

In addition to the imaging results, individuals with highnumeracy were significantly more accurate in estimating the absolute number of filled icons in the NL condition compared to individuals with low-numeracy (see Fig. 5). The results of the NL condition are consistent with a large body of evidence, which has demonstrated a significant positive association between individual differences in numeracy and accuracy in number line estimation. Specifically, an increasing number of behavioural studies with children has demonstrated a reliable and consistent positive association between individual differences in number line performance and individual differences in arithmetic, standardized mathematical achievement test scores and mathematical school grades (Booth and Siegler 2006; Schneider et al. 2009; Siegler and Booth 2004; Thompson and Siegler 2010). In line with these previous findings, the result of the present study demonstrated a more accurate performance during number line estimation in individuals with high-numeracy compared to individuals with lownumeracy. Moreover, the group difference in the number line condition is in line with a study that investigated differences in icon array processing between individuals with low- and high-numeracy (Hess et al. 2011). Results of this study revealed differences in the way icon arrays were processed by individuals with low- and high-numeracy. More specifically, individuals with high-numeracy were shown to extract exact numerical values from icon arrays (i.e., counting the number of icons), whereas individuals with-low numeracy approximated numerical values in icon arrays (i.e., estimation of the numerical values; Hess et al. 2011).

The behavioural mean deviation scores in the HRP condition also differed significantly between the two groups. More specifically, individuals with high-numeracy consistently overestimated (i.e., positive mean deviation scores in relation to the "objective" baseline position of probabilities) health risks compared to their low-numeracy peers. This relative overestimation bias of high-numeracy individuals (i.e., greater deflection from the "objective" baseline position) may not exclusively be explained by differences in numerical abilities, but rather by additional affective mechanisms with which individuals with high-numeracy skills perceived numerical risk information. Previous research has demonstrated that individuals with high-numeracy draw more affective meaning from numbers compared to individuals with low-numeracy, which are more influenced by irrelevant affective considerations (Peters et al. 2006). A tentative explanation of the observed overestimation in individuals with highnumeracy skills may be that individuals with high-numeracy charged numerical risk information with greater emotional valance (hence the greater activation in regions that are associated with emotional processing), which in turn biased risk evaluation in the HRP condition. The low-numeracy individuals, in contrast, may not be extracting the same affective meaning from numerical pictographs as their high-numeracy peers. While this interpretation is appealing, clearly more research is needed to further explore potential individual differences in number processing during health risk perception.

While the present study revealed significant differences between individuals with high-and low-numeracy skills, a limitation of the present interpretation arises form the fact that our numeracy groups were confounded by gender. In order to test this possibility, we performed additional analyses including gender as a between subject factor. These analyses demonstrated no significant influence of gender (all $p$ 's > 0.05; all $\eta^{2}$ 's $<0.01$ ) and as such it is very unlikely that the observed differences in health risk perception are a pure artefact of gender. Nevertheless, it cannot be entirely ruled out that gender exerted some influence on the observed group differences in the present study. Additional studies investigating whether gender indeed exerts any influence during health risk evaluations are needed in the future. In addition, while the SNS has been developed as a tool to predict people's numerical abilities (especially within clinical settings), it should be noted that the SNS is not a substitute for objective and standardized mathematical assessments that measure numerical abilities on a broader scale. Future studies should use such measurements in order to shed additional light on the underlying mechanisms that differentiate individuals with high- and low-numeracy during risk decisions.

In summary, a hallmark of mathematical literacy is the flexible use of numerical information to describe, explain and predict real phenomena and to guide our behaviour in different contextual situations (OECD 2012). The neural mechanisms underlying the translation and integration of numerical information to predict phenomena and to guide behaviour in different contextual situations are not well understood. The present study provides first evidence that the neural correlates associated with health risk decisions differ between individuals with high- and low-numeracy skills. Specifically, the imaging results demonstrated that individuals with high-numeracy engaged regions of the brain, which have been previously associated with emotional processing (e.g., Herwig et al. 2011; Mohr et al. 2010) to a larger extent compared to individuals with low-numeracy skills. This finding does not only demonstrate differential engagement of brain regions between individuals with low- and high-numeracy skills, but also indicates possible differences in the way numerical information are affectively perceived during decision making. Deepening our knowledge of how we process numerical 
information in different contexts has profound implications for educators by helping them to identify those mechanisms that are most likely to add significant value to the translation of numerical information to real life contexts. The present imaging study indicates that affective mechanisms may play a vital role in how we perceive numerical information in emotionally charged situations. Learning to reflect on numerical information, particularly in emotional contexts, may be important for individuals to avoid unfavourable biases during decision-making. Despite these implications, clearly more evidence is needed to better understand the mechanisms at hand to unravel how individuals process numerical information in different contextual situations.

Acknowledgments We thank Daniela Schwab for her assistance in the recruitment of participants and in the fMRI test sessions. In addition, the helpful comments of the anonymous reviewers are gratefully acknowledged.Open Access This article is distributed under the terms of the Creative Commons Attribution 4.0 International License (http://creativecommons.org/licenses/by/4.0/), which permits unrestricted use, distribution, and reproduction in any medium, provided you give appropriate credit to the original author(s) and the source, provide a link to the Creative Commons license, and indicate if changes were made.

\section{Appendix}

Table 4 and Table 5 of the Appendix display all numerical stimuli that were used in the present study.

Table 4 Numerical stimuli used in the HRP condition

\begin{tabular}{llr}
\hline Stimuli & Arrow position & Decade \\
\hline 57 & 98 & 50 \\
48 & 70 & 40 \\
2 & 86 & 0 \\
34 & 49 & 30 \\
69 & 27 & 60 \\
59 & 28 & 50 \\
36 & 56 & 30 \\
87 & 23 & 80 \\
8 & 54 & 0 \\
16 & 52 & 10 \\
27 & 14 & 20 \\
9 & 51 & 0 \\
74 & 13 & 70 \\
82 & 14 & 80 \\
14 & 67 & 10 \\
77 & 67 & 70 \\
22 & 44 & 20 \\
62 & 9 & 60 \\
\hline
\end{tabular}

Table 4 continued

\begin{tabular}{|c|c|c|}
\hline Stimuli & Arrow position & Decade \\
\hline$\overline{28}$ & 89 & 20 \\
\hline 94 & 65 & 90 \\
\hline 43 & 87 & 40 \\
\hline 81 & 10 & 80 \\
\hline 68 & 13 & 60 \\
\hline 85 & 72 & 80 \\
\hline 42 & 20 & 40 \\
\hline 1 & 20 & 0 \\
\hline 63 & 86 & 60 \\
\hline 23 & 40 & 20 \\
\hline 47 & 88 & 40 \\
\hline 95 & 48 & 90 \\
\hline 26 & 10 & 20 \\
\hline 92 & 25 & 90 \\
\hline 12 & 71 & 10 \\
\hline 78 & 1 & 70 \\
\hline 17 & 70 & 10 \\
\hline 65 & 55 & 60 \\
\hline 24 & 59 & 20 \\
\hline 99 & 32 & 90 \\
\hline 83 & 69 & 80 \\
\hline 19 & 8 & 10 \\
\hline 64 & 93 & 60 \\
\hline 55 & 78 & 50 \\
\hline 96 & 54 & 90 \\
\hline 5 & 35 & 0 \\
\hline 38 & 27 & 30 \\
\hline 91 & 52 & 90 \\
\hline 13 & 2 & 10 \\
\hline 45 & 23 & 40 \\
\hline 33 & 67 & 30 \\
\hline 84 & 55 & 80 \\
\hline 71 & 4 & 70 \\
\hline 32 & 79 & 30 \\
\hline 58 & 72 & 50 \\
\hline 39 & 2 & 30 \\
\hline 56 & 22 & 50 \\
\hline 79 & 26 & 70 \\
\hline 46 & 15 & 40 \\
\hline 53 & 42 & 50 \\
\hline 6 & 34 & 0 \\
\hline 72 & 62 & 70 \\
\hline
\end{tabular}

The column "Stimuli" represents the number of filled icons (i.e., black diamonds) in the pictographs 
Table 5 Numerical stimuli used in the NL condition

\begin{tabular}{|c|c|c|}
\hline Stimuli & Arrow position & Decade \\
\hline 95 & 33 & 90 \\
\hline 24 & 82 & 20 \\
\hline 34 & 66 & 30 \\
\hline 59 & 44 & 50 \\
\hline 19 & 42 & 10 \\
\hline 99 & 63 & 90 \\
\hline 55 & 9 & 50 \\
\hline 69 & 54 & 60 \\
\hline 89 & 99 & 80 \\
\hline 29 & 54 & 20 \\
\hline 74 & 33 & 70 \\
\hline 85 & 32 & 80 \\
\hline 44 & 58 & 40 \\
\hline 38 & 48 & 30 \\
\hline 9 & 20 & 0 \\
\hline 65 & 17 & 60 \\
\hline 4 & 79 & 0 \\
\hline 79 & 9 & 70 \\
\hline 14 & 1 & 10 \\
\hline 49 & 3 & 40 \\
\hline 77 & 31 & 70 \\
\hline 27 & 10 & 20 \\
\hline 62 & 91 & 60 \\
\hline 12 & 28 & 10 \\
\hline 93 & 15 & 90 \\
\hline 32 & 86 & 30 \\
\hline 46 & 86 & 40 \\
\hline 36 & 9 & 30 \\
\hline 57 & 80 & 50 \\
\hline 53 & 23 & 50 \\
\hline 82 & 27 & 80 \\
\hline 2 & 53 & 0 \\
\hline 42 & 25 & 40 \\
\hline 87 & 21 & 80 \\
\hline 7 & 82 & 0 \\
\hline 72 & 61 & 70 \\
\hline 97 & 75 & 90 \\
\hline 16 & 47 & 10 \\
\hline 67 & 24 & 60 \\
\hline 22 & 90 & 20 \\
\hline
\end{tabular}

The column "Stimuli" represents the number of filled icons (i.e., black diamonds) in the pictographs

\section{References}

Ansari, D. (2008). Effects of development and enculturation on number representation in the brain. Nature Reviews Neuroscience, 9(4), 278-291.
Ansari, D., Garcia, N., Lucas, E., Hamon, K., \& Dhital, B. (2005). Neural correlates of symbolic number processing in children and adults. NeuroReport, 16(16), 1769-1773.

Ansari, D., \& Vogel, S. E. (2013). Cognitive neuroscience of numerical cognition. In K. Ochsner \& S. M. Kosslyn (Eds.), Oxford handbook of cognitive neuroscience (Vol. 2, pp. 382-400). London: Oxford University Press.

Apter, A. J., Paasche-Orlow, M. K., Remillard, J. T., Bennett, I. M., Ben-Joseph, E. P., Batista, R. M., et al. (2008). Numeracy and communication with patients: they are counting on us. Journal of General Internal Medicine, 23(12), 2117-2124.

Bach, D. R., Seymour, B., \& Dolan, R. J. (2009). Neural activity associated with the passive prediction of ambiguity and risk for aversive events. The Journal of Neuroscience, 29(6), 1648-1656.

Booth, J. L., \& Siegler, R. S. (2006). Developmental and individual differences in pure numerical estimation. Developmental Psychology, 42(1), 189-201.

Cavanna, A. E., \& Trimble, M. R. (2006). The precuneus: A review of its functional anatomy and behavioural correlates. Brain, 129(3), 564-583.

Dehaene, S., Piazza, M., Pinel, P., \& Cohen, L. (2003). Three parietal circuits for number processing. Cognitive Neuropsychology, 20(3), 487-506.

Fagerlin, A., Wang, C., \& Ubel, P. A. (2005). Reducing the influence of anecdotal reasoning on people's health care decisions: Is a picture worth a thousand statistics? Medical Decision Making, 25(4), 398-405.

Fagerlin, A., Zikmund-Fisher, B. J., Ubel, P. A., Jankovic, A., Derry, H. A., \& Smith, D. M. (2007). Measuring numeracy without a math test: Development of the Subjective Numeracy Scale. Medical Decision Making, 27, 672-680.

Feigenson, L., Libertus, M. E., \& Halberda, J. (2013). Links between the intuitive sense of number and formal mathematics ability. Child Development Perspectives, 7(2), 74-79.

Feldman-Stewart, D., Kocovski, N., McConnell, B. A., Brundage, M. D., \& Mackillop, W. J. (2000). Perception of quantitative information for treatment decisions. Medical Decision Making, 20(2), 228-238.

Galesic, M., Garcia-Retamero, R., \& Gigerenzer, G. (2009). Using icon arrays to communicate medical risks: overcoming low numeracy. Health Psychology, 28(2), 210-216.

Garcia-Retamero, R., \& Galesic, M. (2010). Who profits from visual aids: Overcoming challenges in people's understanding of risks. Social Science and Medicine, 70(7), 1019-1025.

Gigerenzer, G., \& Edwards, A. (2003). Simple tools for understanding risks: From innumeracy to insight. BMJ, 327(7417), 741-744.

Gilmore, A. W., Nelson, S. M., \& McDermott, K. B. (2015). A parietal memory network revealed by multiple MRI methods. Trends in Cognitive Sciences, 19(9), 534-543.

Grabner, R. H., Ansari, D., Reishofer, G., Stern, E., Ebner, F., \& Neuper, C. (2007). Individual differences in mathematical competence predict parietal brain activation during mental calculation. NeuroImage, 38(2), 346-356.

Heekeren, H. R., Marrett, S., \& Ungerleider, L. G. (2008). The neural systems that mediate human perceptual decision making. Nature Reviews Neuroscience, 9(6), 467-479.

Herwig, U., Brühl, A. B., Viebke, M. C., Scholz, R. W., Knoch, D., \& Siegrist, M. (2011). Neural correlates of evaluating hazards of high risk. Brain Research, 1400, 78-86.

Hess, R., Visschers, V. H. M., \& Siegrist, M. (2011). Risk communication with pictographs: The role of numeracy and graph processing. Judgment and Decision Making, 6(3), 263-274.

Hoffrage, U., Lindsey, S., Hertwig, R., \& Gigerenzer, G. (2000). Medicine. Communicating statistical information. Science (New York, N.Y.), 290(5500), 2261-2262. 
Holloway, I. D., \& Ansari, D. (2009). Mapping numerical magnitudes onto symbols: The numerical distance effect and individual differences in children's mathematics achievement. Journal of Experimental Child Psychology, 103(1), 17-29.

Holloway, I. D., Battista, C., Vogel, S. E., \& Ansari, D. (2012). Sematic and perceptual processing of number symbols: Evidence from a cross-linguistic fMRI adaptation sudy. Journal of Cognitive Neuroscience, 25(3), 388-400.

Holloway, I. D., Price, G. R., \& Ansari, D. (2010). Common and segregated neural pathways for the processing of symbolic and nonsymbolic numerical magnitude: An fMRI study. NeuroImage, 49(1), 1006-1017.

Huettel, S. A., Song, A. W., \& Mccarthy, G. (2008). Functional magnetic resonance imaging ( 2 nd ed.). Sunderland: Sinauer Associates, Inc.

Kaufmann, L., Vogel, S. E., Starke, M., Kremser, C., \& Schocke, M. (2009). Numerical and non-numerical ordinality processing in children with and without developmental dyscalculia: Evidence from fMRI. Cognitive Development, 24(4), 486-494.

Knight, F. H. (1921). Risk, uncertainty and profit. Boston: Houghton Mifflin.

Lipkus, I. M., \& Peters, E. (2009). Understanding the role of numeracy in health: Proposed theoretical framework and practical insights. Health Education \& Behavior, 36(6), 1065-1081.

Lipkus, I., \& Peters, E. (2010). Breast cancer patients' treatment expectations after exposure to the decision aid program adjuvant online: The influence of numeracy. Medical Decision Making, 30(4), 464-473.

Lipkus, I. M., Samsa, G., \& Rimer, B. K. (2001). General performance on a numeracy scale among highly educated samples. Medical Decision Making, 21, 37-44.

Mohr, P. N. C., Biele, G., \& Heekeren, H. R. (2010). Neural processing of risk. The Journal of Neuroscience, 30(19), 6613-6619.

Nelson, W., Reyna, V. F., Fagerlin, A., Lipkus, I., \& Peters, E. (2008). Clinical implications of numeracy: Theory and practice. Annals of Behavioral Medicine, 35(3), 261-274.

Nieder, A., \& Dehaene, S. (2009). Representation of number in the brain. Annual Review of Neuroscience, 32, 185-208.

OECD. (2012). PISA 2012 mathematics framework to OECD. Paris: OECD Publications.

Peters, E. (2008). Numeracy and the perception and communication of risk. Annals of the New York Academy of Sciences, 1128, 1-7.

Peters, E., Västfjäll, D., Slovic, P., Mertz, C. K., Mazzocco, K., \& Dickert, S. (2006). Numeracy and decision making. Psychological Science, 17(5), 407-413.

Pinel, P., Dehaene, S., Rivière, D., \& LeBihan, D. (2001). Modulation of parietal activation by semantic distance in a number comparison task. NeuroImage, 14(5), 1013-1026.

Pinel, P., Le Clec'H, G., van de Moortele, P. F., Naccache, L., Le Bihan, D., \& Dehaene, S. (1999). Event-related fMRI analysis of the cerebral circuit for number comparison. Neuroreport, 10(7), $1473-1479$.

Price, G. R., Holloway, I., Räsänen, P., Vesterinen, M., \& Ansari, D. (2007). Impaired parietal magnitude processing in developmental dyscalculia. Current Biology, 17(24), R1042-R1043.

Quartz, S. R. (2009). Reason, emotion and decision-making: Risk and reward computation with feeling. Trends in Cognitive Sciences, 13(5), 209-215.
Ridderinkhof, K. R., Ullsperger, M., Crone, E. A., \& Nieuwenhuis, S. (2004). The role of the medial frontal cortex in cognitive control. Science (New York, N.Y.), 306(5695), 443-447.

Schneider, M., Grabner, R. H., \& Paetsch, J. (2009). Mental number line, number line estimation, and mathematical achievement: Their interrelations in grades 5 and 6. Journal of Educational Psychology, 101(2), 359-372.

Siegler, R. S., \& Booth, J. L. (2004). Development of numerical estimation in young children. Child Development, 75(2), 428-444.

Simon, O., Cohen, L., Bihan, D. Le, \& Dehaene, S. (2002). Topographical layout of hand, eye, calculation, and language-related areas in the human parietal lobe. Neuron, 33(3), 475-487.

Simon, O., Kherif, F., Flandin, G., Poline, J.-B., Rivière, D., Mangin, J.-F., et al. (2004). Automatized clustering and functional geometry of human parietofrontal networks for language, space, and number. NeuroImage, 23(3), 1192-1202.

Tanius, B. E., Wood, S., Hanoch, Y., \& Rice, T. (2009). Aging and choice: Applications to medicare part D. Judgment and Decision Making, 4(1), 92-101.

Thompson, C. A., \& Siegler, R. S. (2010). Linear numerical-magnitude representations aid children's memory for numbers. Psychological Science, 21(9), 1274-1281.

Venkatraman, V., Payne, J. W., Bettman, J. R., Luce, M. F., \& Huettel, S. A. (2009). Separate neural mechanisms underlie choices and strategic preferences in risky decision making. Neuron, 62(4), 593-602.

Visschers, V. H. M., Meertens, R. M., Passchier, W. W. F., \& de Vries, N. N. K. (2009). Probability information in risk communication: A review of the research literature. Risk Analysis, 29(2), 267-287.

Vogel, S. E., \& Ansari, D. (2012). Neurocognitive foundations of typical and atypical number processing. Lernen Und Lernstoerungen, 1(2), 135-149.

Vogel, S. E., Celia, G., \& Ansari, D. (2015). Developmental specialization of the left parietal cortex for the semantic representation of Arabic numerals: An fMR-Adaptaton study. Developmental Cognitive Neuroscience, 12, 61-73.

Vogel, S. E., Grabner, R. H., Schneider, M., Siegler, R. S., \& Ansari, D. (2013). Overlapping and distinct brain regions involved in estimating the spatial position of numerical and non-numerical magnitudes: An fMRI study. Neuropsychologia, 51(5), 979-989.

Vorhold, V., Giessing, C., Wiedemann, P. M., Schütz, H., Gauggel, S., \& Fink, G. R. (2007). The neural basis of risk ratings: Evidence from a functional magnetic resonance imaging (fMRI) study. Neuropsychologia, 45(14), 3242-3250.

Xu, L., Liang, Z. Y., Wang, K., Li, S., \& Jiang, T. (2009). Neural mechanism of intertemporal choice: From discounting future gains to future losses. Brain Research, 1261, 65-74.

Zikmund-Fisher, B. J., Fagerlin, A., \& Ubel, P. A. (2008). Improving understanding of adjuvant therapy options by using simpler risk graphics. Cancer, 113(12), 3382-3390.

Zikmund-Fisher, B. J., Smith, D. M., Ubel, P. A., \& Fagerlin, A. (2007). Validation of the Subjective Numeracy Scale: Effects of low numeracy on comprehension of risk communications and utility elicitations. Medical Decision Making, 27, 663-671. 